\title{
Intense Muon Physics Working Group Summary
}

\author{
B. Lee Roberts ${ }^{\mathrm{a}}$ Marco Grassi ${ }^{\mathrm{b}}$ Akira Sato $^{\mathrm{c}}$ \\ ${ }^{a}$ Department of Physics \\ Boston University \\ Boston, MA 02215 USA \\ ${ }^{\mathrm{b}}$ INFN, Pisa \\ ${ }^{\mathrm{c}}$ Dept. of Physics \\ Osaka University \\ Toyonaka, Osaka, Japan 560-0043
}

The intense muon beams which will be available at a neutrino factory provide a unique opportunity for searching for physics beyond the standard model, both in lepton flavor violation and in the search for a permanent electric dipole moment for the muon. Other experiments which can use intense muon beams will also be possible.

\section{Introduction}

Over many years, the muon has provided important input to the standard model: the value of the weak coupling constant $G_{F}$, strong proof of the $V-A$ nature of the weak interaction, information on the induced weak pseudoscalar form factor $g_{p}$, strong contraints on new physics from its anomalous magnetic moment, and sensitive limits on the presence of new physics which would cause lepton flavor violation in the muon's decay. In addition to its contributions to particle physics, the muon has become a useful tool in condensed matter physics.

We heard about all of these topics in our working group sessions, and the projections from these very nice talks are on the NuFact website. In this summary we will focus on the "muon trio": the anomalous magnetic dipole moment (MDM) [1] $a_{\mu}=(g-2) / 2$; the search for a permanent electric dipole moment (EDM) of the muon 2] which would signify $\not P, T$ and by implication $C P$ violation if $C P T$ is valid; and the search for lepton flavor violation $(L F V) 345$. We focus on these experiments, and specifically on the latter two because they require the highest possible muon flux, which would only be available at a high-intensity muon source such as a neutrino factory. Useful reviews can be found for: muon physics at a neutrio factory $[\underline{6}$; theory of muon $(g-2)[78]$; electric dipole moments [9]; and lepton flavor violation [10 11].

The muon anomalous magnetic moment has now been measured by BNL E821 to a relative precision of 0.5 parts per million (ppm), 12 1314] and it is proposed to improve this experiment to $0.2 \mathrm{ppm}$ in an upgraded experiment E969 at Brookhaven. E969 has scientific approval but is not yet funded 15. Since the first precise result from E821 became available 12, there has been an approximate 2.5 standard deviation discrepancy between theory (presently known to about $0.6 \mathrm{ppm}$ ) and experiment, when the hadronic contribution is taken from $e^{+} e^{-}$data. There is much activity worldwide in improving our knowledge of the hadronic contribution 1617. This increased precision available to E969, combined with the expected improvements in the knowledge of the hadronic contribution, eventually should give at least a factor of two reduction in the combined experiment-theory uncertainty when comparing the two. It is possible to improve on the experiment further 18, but to fully realize the potential of the improved experimental measurement, the hadronic contribution would need to be known 
below $0.1 \mathrm{ppm}$ uncertainty.

One of the most important roles the measurements of $a_{\mu}$ have played in the past is placing serious restrictions on physics beyond the standard model [7]. With the development of supersymmetric theories as a favored scheme of physics beyond the standard model, interest in the experimental and theoretical value of $a_{\mu}$ has grown substantially. SUSY contributions to $a_{\mu}$ could be at a measurable level in a broad range of models. It is interesting, although speculative, to note that there is a certain consistency of evidence supporting the proposal that the dark matter candidate is the lightest supersymmetric partner, as shown in Fig. [19]. The projected $(g-2)$ precision of E969 plus the expected improved theory uncertainty would reduce the size of the $2 \sigma$ band of allowed values down to the present $1 \sigma$ band shown in Fig. [1]

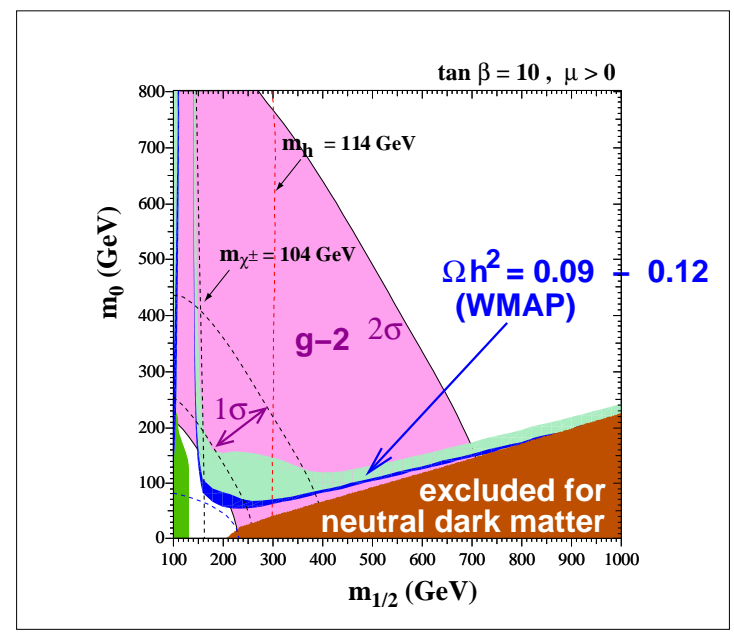

Figure 1. Limits on dark matter placed by various inputs in CMSSM. The $\Delta$ between the standard model and the E821 value of $(g-2)$ is $(24 \pm 10) \times 10^{-10}$, obtained from the $e^{+} e^{-}$based theory evaluation of Ref. [7. $m_{0}$ and $m_{1 / 2}$ are the scalar mass and gaugino mass respectively. (Courtesy of K. Olive)

Furthermore, there is a complementarity be- tween the SUSY contributions to the MDM, EDM and transition moment for the lepton-flavor violating process $\mu^{-} \rightarrow e^{-}$in the field of a nucleus. The MDM and EDM are related to the real and imaginary parts of the diagonal element of the slepton mixing matrix, and the transition moment is related to the off diagonal one, as shown in Fig. 2

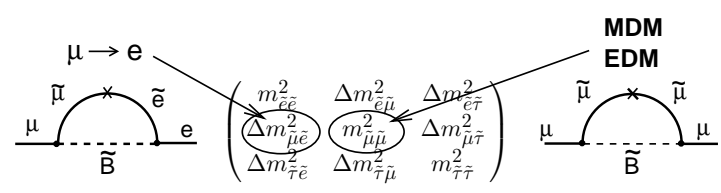

Figure 2. The supersymmetric contributions to the anomaly, and to $\mu \rightarrow e$ conversion, showing the relevant slepton mixing matrix elements. The MDM and EDM give the real and imaginary parts of the matrix element respectively.

While magnetic dipole moments (MDMs) are a natural property of charged particles with spin, electric dipole moments (EDMs) are forbidden both by parity $(P)$ and by time reversal $(T)$ symmetries 222324 . This can be seen by examining the Hamiltonian for a spin one-half particle in the presence of both an electric and magnetic field, $\mathcal{H}=-\vec{\mu} \cdot \vec{B}-\vec{d} \cdot \vec{E}$. The transformation properties of $\vec{E}, \vec{B}, \vec{\mu}$ and $\vec{d}$ are given in Table 1 and we see that while $\vec{\mu} \cdot \vec{B}$ is even under all three, $\vec{d} \cdot \vec{E}$ is odd under both $P$ and $T$. Thus the existence of an EDM implies that both $P$ and $T$ are violated. In the context of CPT symmetry, an EDM implies $C P$ violation. The standard model value for the electron and muon EDMs are well beyond the reach of experiment (see Table 2), so observation of a non-zero $e$ or $\mu \mathrm{EDM}$ would be a clear signal for new physics. Since the presently known $\mathrm{CP}$ violation is inadequate to describe the baryon asymmetry in the universe, additional sources of $C P$ violation should be present. Furthermore, we do expect to find $C P$ violation in the lepton sector. New dynamics such as supersymmetry could 
easily produce new sources of $C P$ violation which could have a possible connection with cosmology (leptogenesis) 2512627.

\begin{tabular}{cccc}
\hline & $\vec{E}$ & $\vec{B}$ & $\vec{\mu}$ or $\vec{d}$ \\
\hline$P$ & - & + & + \\
$C$ & - & - & - \\
$T$ & + & - & - \\
\hline
\end{tabular}

Table 1

Transformation properties of the magnetic and electric fields and dipole moments. The dipole moments are assumed to be along the spin vector, and to consist of charge times spin in the appropriate units.

\begin{tabular}{ccc}
\hline Particle & $\begin{array}{c}\text { Present Limit } \\
(\mathrm{e}-\mathrm{cm})\end{array}$ & $\begin{array}{c}\text { SM Value } \\
(\mathrm{e}-\mathrm{cm})\end{array}$ \\
\hline $\mathrm{n}[\mathrm{30}]$ & $6.3 \times 10^{-26}$ & $\sim 10^{-31}$ \\
\hline$e^{-}[31]$ & $\sim 1.6 \times 10^{-27}$ & $<10^{-41}$ \\
\hline$\mu[29]$ & $<10^{-18}(\mathrm{CERN})$ & $<10^{-38}$ \\
& $\sim 10^{-19}(\mathrm{E} 821)^{*}$ & \\
& $\sim 10^{-24} \mathrm{~J}-P A R C^{\dagger}$ \\
\hline
\end{tabular}

Table 2

Measured limits on electric dipole moments, and their standard model values.

* Estimated limit, work in progress.

${ }^{\dagger}$ Letter of Intent (LOI) to J-PARC for a new dedicated experiment 32 .

The possiblity of an experiment to search for a permanent EDM of the muon with a design sensitivity of $10^{-24} e-\mathrm{cm}$ is being studied, either for JPARC or another high intensity muon source 32]. This sensitivity lies well within values predicted by some SUSY models 27. Feng, et al.28, have calculated the range of $\phi_{C P}$ available to such an experiment, assuming a new physics contribution to $a_{\mu}$ of $3 \times 10^{-9}$,

$d_{\mu}^{\mathrm{NP}} \simeq 3 \times 10^{-22}\left(\frac{a_{\mu}^{\mathrm{NP}}}{3 \times 10^{-9}}\right) \tan \phi_{C P} e-\mathrm{cm},(1)$ where $\phi_{C P}$ is a $C P$ violating phase. This range is shown in Fig. [3]

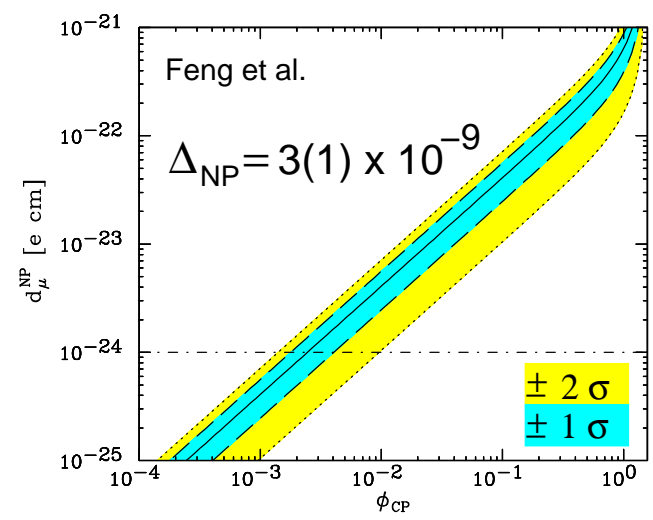

Figure 3. The range of $\phi_{C P}$ available to a dedicated muon EDM experiment 28. The two bands show the one and two standard-deviation ranges if $a_{\mu}$ differs from the standard model value by $(3 \pm 1) \times 10^{-9}$.

Of course, if any EDM is observed, one wishes to measure as many other EDMs as possible to understand the nature of the interaction. While naively the magnitude of the muon and electron EDMs scale linearly with mass, in some theories the muon EDM is greatly enhanced compared to linear scaling from the electron EDM, when the heavy neutrinos of the theory are nondegenerate 25 26].

The lepton flavor violation processes

$$
\begin{aligned}
\mu^{+} & \rightarrow e^{+} \gamma \\
\mu^{+} & \rightarrow e^{+} e^{+} e^{-} \\
\mu^{-} N & \rightarrow e^{-} N \\
\mu^{+} e^{-} & \rightarrow \mu^{-} e^{+}
\end{aligned}
$$

are forbidden in the standard model.

In a large class of models, if the $\Delta \ell=1 L F V$ decay goes through the transition magnetic mo- 
ment, one finds 6

$\frac{B(\mu N \rightarrow e N)}{B(\mu \rightarrow e \gamma)}=2 \times 10^{-3} B(A, Z)$,

where $B(A, Z)$ is a coefficient of order 1 for nuclei heavier than aluminum 20 . For other models, these two rates can be the same [6], so in the design of new experiments the reach in single event sensitivity for the coherent muon conversion experiments needs to be several orders of magnitude smaller than for $\mu \rightarrow e \gamma$ to probe the former class of models with equal sensitivity. Connections between $L F V$ and neutrino oscillations have been explored in several papers 1121 .

The experimental history of searches for $L F V$ can be seen in Fig. 4 Only coherent muon conversion does not require coincidence measurements. The decay $\mu \rightarrow 3 e$, while theoretically appealing, requires a triple coincidence and sensitivity to the whole phase space of the decay, and thereby is experimentally more challenging.

\section{Searches for Lepton Number Violation}

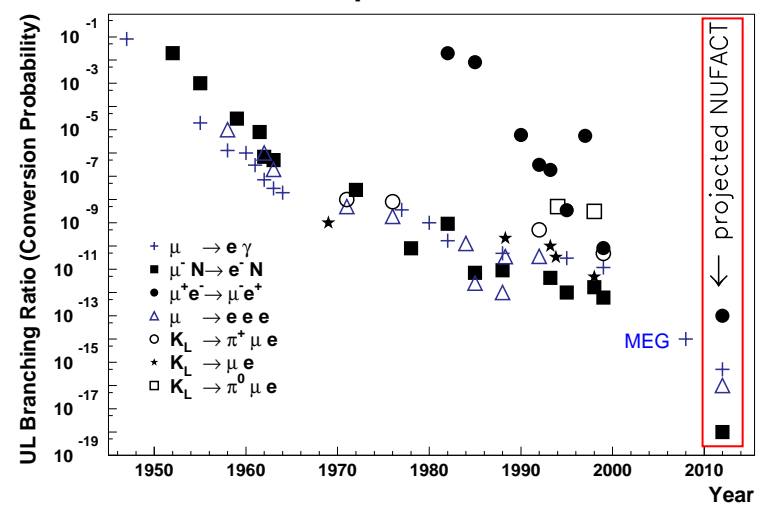

Figure 4. Experimental limits on lepton flavor violation. The future projections include the MEG experiment's limit, and potential limits which could be reached at the front end of a neutrino factory. These future projections are taken from Ref. 6]
The muonium to antimuonium conversion (process (4) above) 33 and shown in Fig. 5] represents a change of two units of lepton number, analogous to $K^{0} \bar{K}^{0}$ oscillations, as originally proposed by Pontecorvo 34, $\Delta \ell=2$, and the single event sensitivity obtained 35 . was $P_{M \bar{M}}=$ $8.2 \times 10^{-11}$ which implies a coupling $G_{M \bar{M}} \leq$ $3 \times 10^{-3} G_{F}$ at $90 \%$ C.L., where $G_{F}$ is the Fermi coupling constant. A broad range of speculative theories such as left-right symmetry, R-parity violating supersymmetry, etc. 36, could permit such an oscillation.

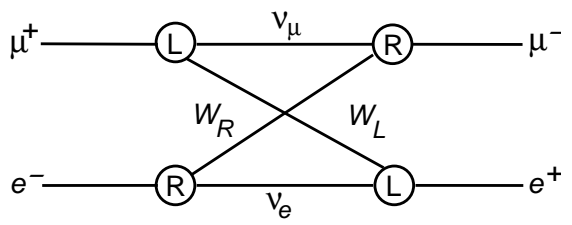

Figure 5. Muonium to antiMuonium conversion in left-right symmetric models with heavy Majorana neutrinos.

Also mentioned in our session was the possibility to search for the $L F V$ reaction $\mu N \rightarrow \tau X$ and $e N \rightarrow \tau X$ in deep inelastic scattering 37 .

\section{Experimental Considerations}

\subsection{Static Magnetic and Electric Dipole Moments}

The muon $(g-2)$ experiment stores muons in a magnetic storage ring with a very uniform magnetic field $( \pm 1 \mathrm{ppm}$ uniformity averaged over azimuth) with electric quadrupoles for vertical focusing. For a muon traveling in a plane transverse to the magnetic field the spin precesses relative to the momentum with the frequency

$\vec{\omega}_{a}=-\frac{e}{m c}\left[a_{\mu} \vec{B}-\left(a_{\mu}-\frac{1}{\gamma^{2}-1}\right) \vec{\beta} \times \vec{E}\right]$,

The experiment is operated at the "magic" $\gamma=$ 29.3 at which an electric field does not contribute 
to the spin motion relative to the momentum. For muons with $\gamma=29.3$ in an electric field alone, the spin would remain along the momentum vector.

If an electric dipole moment is present, the spin precession relative to the momentum is given by

$$
\begin{aligned}
\vec{\omega} & =-\frac{e}{m}\left[a_{\mu} \vec{B}-\left(a_{\mu}-\frac{1}{\gamma^{2}-1}\right) \frac{\vec{\beta} \times \vec{E}}{c}\right] \\
& +\frac{e}{m}\left[\frac{\eta}{2}\left(\frac{\vec{E}}{c}+\vec{\beta} \times \vec{B}\right)\right]=\vec{\omega}_{a}+\vec{\omega}_{\eta}
\end{aligned}
$$

where

$d_{\mu}=\frac{\eta}{2}\left(\frac{e \hbar}{2 m c}\right) \simeq \eta \times 4.7 \times 10^{-14} e-\mathrm{cm}$

and $a_{\mu}=(g-2) / 2$. For $\beta \simeq 1$, the motional electric field $\vec{\beta} \times \vec{B}$ is much larger $(\sim \mathrm{GV} / \mathrm{m})$ than electric fields which can be obtained in the laboratory, and the two vector frequencies are orthogonal to each other as illustrated in Fig. [6]

The EDM has two effects on the precession: the magnitude of the observed frequency is increased, and the precession plane is tipped relative to the magnetic field. E821 was operated at the magic $\gamma$ so that the focusing electric field did not cause a spin precession. In E821 the tipping of the precession plane is very small, $\left(\eta / 2 a_{\mu} \simeq 9 \mathrm{mrad}\right)$ if one uses the CERN EDM limit 29 given in Table 2 This small tipping angle makes it very difficult to observe an EDM effect in E821, since the $(g-2)$ precession $\left(\omega_{a}\right)$ is such a large effect.

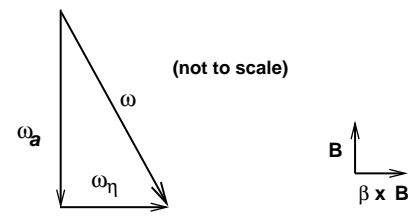

Figure 6. A sketch showing the relationship between $\omega_{a}$ and $\omega_{\eta}$.

The experimental signal for the MDM and EDM measurements is the $e^{ \pm}$from $\mu^{ \pm}$decay.
The time and energy of each event is stored for analysis offline. Muon decay is a three-body decay, so the $3.1 \mathrm{GeV}$ muons produce a continuum of positrons (electrons) from the end-point energy down. Since the highest energy $e^{ \pm}$are correlated with the muon spin, if one counts high-energy $e^{ \pm}$as a function of time, one gets an exponential from muon decay modulated by the $(g-2)$ precession 121314 .

We have recently introduced a new idea which optimizes the EDM signal by operating a new dedicated storage ring off of the magic momentum, and uses a radial electric field to turn off the $(g-2)$ precession. Then the spin will follow the momentum as the muons go around the ring, except for any movement arising from an EDM 38. The dedicated experiment will be operated well off of the magic $\gamma$, for example $\gamma=5$ and $p_{\mu}=500 \mathrm{MeV} / \mathrm{c}$. The EDM would cause a steady build-up of the spin out of the plane with time. Detectors would be placed above and below the storage region, and a time-dependent up-down asymmetry $R$ would be the signal of an EDM,

$R=\frac{N_{\text {up }}-N_{\text {down }}}{N_{\text {up }}+N_{\text {down }}}$.

The figure of merit for statistics in the EDM experiment is the number of muons times the polarization squared. In order to reach $10^{-24} e \mathrm{~cm}$, the muon EDM experiment would need $N P^{2} \simeq$ $5 \times 10^{16}$, a number only available at a future facility. While progress can still be made at Brookhaven on $a_{\mu}$, a dedicated muon EDM experiment must be done elsewhere.

\section{2. $\mu^{+} \rightarrow e \gamma$ and $\mu^{-} N \rightarrow e^{-} N$}

From the experimental side, the "next generation" $\mu \rightarrow e \gamma$ experiment, MEG, is now under construction at PSI [3, and data collection is to begin in 2006. Since the decay occurs at rest, the photon and positron are back-to-back, and share the energy of the muon mass. This experiment makes use of a unique "COBRA" magnet which produces a constant bending radius for the monoenergetic $e^{+}$independent of its angle. The photon is detected by a large liquid Xe scintillation detector as shown in Fig. 7 The MEG experiment 
should probe down to the $10^{-14}$ level. Because this is a coincidence experiment, it is difficult to go to rates higher than will be used at PSI for MEG, without major changes in the technology for the detection of the photon and electron.
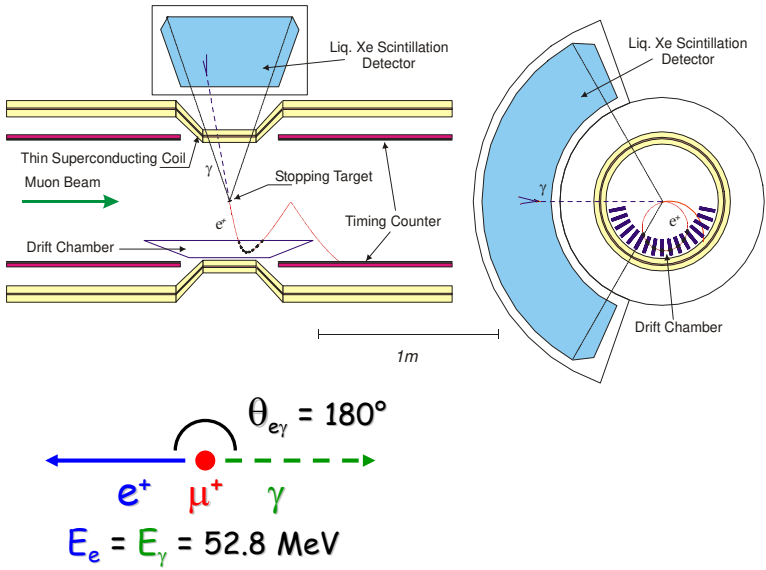

Figure 7. The end and side views of the MEG experiment. Since the muon is at rest, the photon and positron are at $180^{\circ}$. The positron is tracked in a magnetic field which produces a constant bending radius, independent of angle.

The LFV experiment which holds the most promise to go to higher muon rates, is the coherent conversion of a muon to an electron in the field of a nucleus, $\mu^{-}+N \rightarrow e^{-}+N$. The signal is a single electron with an energy equal to the muon mass, less the binding energy of the muon in the $1 \mathrm{~S}$ state of the muonic atom. The MECO experiment at Brookhaven had proposed to place a pion production target inside of a graded solenoidal field, which captures $\pi^{-}$with high efficiency, and then transport them through a long solenoid where the charge separation is done, and then onto a stopping target where the conversion takes place, as shown in Fig. 8] Unfortunately MECO 4, which projected a singleevent sensitivity of $2 \times 10^{-17}$, was terminated in
August 2005.

A $\mu-e$ conversion experiment, PRIME [5], is also being proposed for J-PARC but is not yet funded. Prime uses the same production target and transport solenoid scheme, but uses a FFAG ring to phase rotate the muon beam, 39] to dramatically decrease its momentum spread. This scheme has the additional benefit that all pions decay during the storage time, thus effectively reducing the possibility of backgrounds from pion capture to a completely negligible level. The hope of such an experiment when coupled with a sufficiently strong muon source might be a few $10^{-19}$ single event sensitivity.

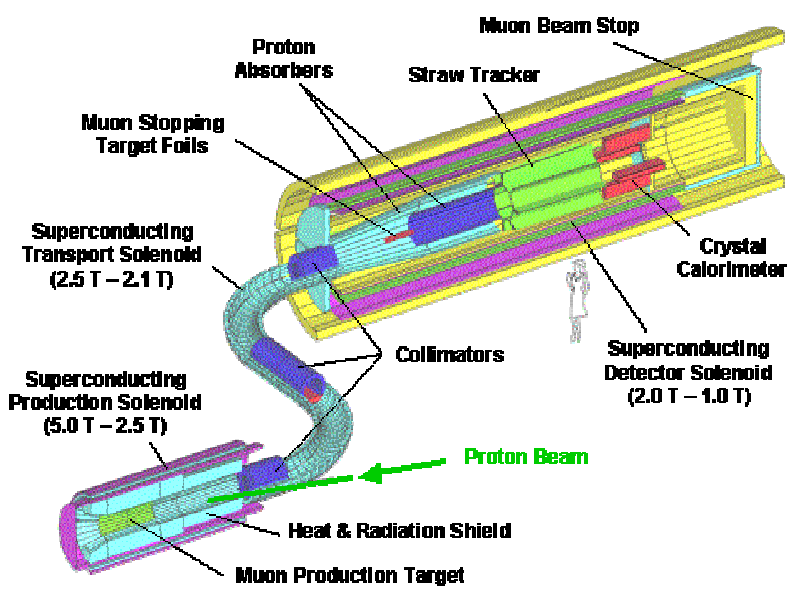

Figure 8. The MECO experiment. The production target is in a superconducting solenoid which has a graded magnetic field, such that pions which go away from the transport solenoid are reflected back and towards the transport solenoid.

With a pulsed beam, and a low background environment, a muonium to anti muonium experiment could also be possible at a high-intensity muon source. The signal of the decay of the $\bar{M}=e^{+} \mu^{-}$is a slow positron which is stopped in a multichannel plate and then annihilates producing two back-to-back $0.511 \mathrm{MeV}$ gamma rays, 
plus a slow muon which decays to an energetic electron.

\section{Other Muon Experiments}

In our working group we heard two very interesting talks on the $\mu$ Lan and MuCap experiments now in progress at PSI 40 to measure the muon lifetime $\left(G_{F}\right)$ and the $\mu^{-}$capture rate in hydrogen to measure the induced pseudoscalar form factor $g_{p}$.

An intense muon beam has a several interesting applications besides those in particle physics. We heard two talks on the use of muons in condensed matter physics. 4142 One concerned studies on the origin of n-type conductivity in wide gap semiconductors. Isolated hydrogen centers in semiconductors are one of the candidates to explain the origin of this effect. Muon Spin Rotation/Relaxation, $\mu \mathrm{SR}$, is a powerful tool for studies of this subject. Data taken at the KEK Muon Science Laboratory and at TRIUMF were presented.

A very low-energy polarized $\mu^{+}$beam is also useful for nanoscience. Epithermal muons, which have the peak energy of $15 \pm 10 \mathrm{eV}$ and are $100 \%$ polarized, enable us to make nano-scale depth resolved $\mu \mathrm{SR}$ measurements in near surface regions of materials. Among the examples using a PSI epithermal muon beam was a very nice measurement of the magnetic penetration depth into a superconducting sample (Meissner effect). A new high intensity surface muon beam line at PSI with seven times the present intensity, along with an upgrade to the present apparatus, is now in the commissioning phase.

Another topic discussed was muon catalyzed fusion $(\mu \mathrm{CF}) 43$. In $\mu \mathrm{CF}$, a muon serves as a catalyst to enhance the deuterium fusion rate through the resonant formation of a muonic molecule such as a $d d \mu$. Experiments were carried out with normal- and ortho- $\mathrm{D}_{2}$ in solid, liquid and gas states, and a clear effect of the orthopara ratio for $d d \mu$ formation in $\mathrm{D}_{2}$ was observed. This is the most important rate-limiting process in $\mu \mathrm{CF}$ 44]. A result of theoretical calculations, which predicted an enhanced effect for $d \mu \tau$ formation, opens up the possibility for enhancement of $\mu \mathrm{CF}$ by controlling initial molecular states.

\section{Summary and Conclusions}

The questions addressed by muon physics are at the center of the field of particle physics. There is an important program of muon physics which will be possible at the front-end of a $\nu$ factory which makes use of the very intense flux which will be available there. If such a muon facility exists, there will also be a program of other very interesting muon experiments which is possible.

We wish to thank all our colleagues for coming to our working group and presenting us with such an interesting program of muon physics.

\section{REFERENCES}

1. B.L. Roberts, at this meeting.

2. G. Onderwater, at this meeting.

3. The MEG $\left(\mu^{+} \rightarrow e^{+} \gamma\right)$ Experiment at the Paul Scherrer Institute, F. Gatti at this meeting, A. Baldini and T. Mori spokespersons. http://meg.web.psi.ch/

4. The MECO experiment,M. Aoiki at this meeting, W. Molzon, spokesperson.

5. A. Sato at this meeting, and J-PARC Letter of Intent L25: An Experimental Search for the $\mu^{-} \rightarrow e^{-}$Conversion Process at an Ultimate Sensitivity of the order of $10^{-18}$ with PRISM, Contact persons: Y. Mori, K. Yoshimura, N. Sasao, Y. Kuno.

6. J. Äysto, et al., hep-ph/0109217. This report of a CERN working group lays out a nice case for muon physics at a neutrino factory.

7. M. Davier and W. Marciano, Ann. Rev. Nucl. Part. Sci. 54 (2004) 115, and references therein.

8. M. Passera, at this meeting and J. Phys. G 31 (2005), R75.

9. Maxim Pospelov and Adam Ritz, Ann. of Phys. 318 (2005) 119.

10. Yoshitaka Kuno and Yasuhiro Okada, Rev. Mod. Phys. Phys. 73 (2001) 151, and references therein.

11. Antonio Masiero, Sudhir K. Vempati and Oscar Vieves, New. J. Phys. 6 (2004) 421, hep-ph/0407325v2, and references therein. 
12. H.N. Brown, et al., (Muon $(g-2)$ Collaboration), Phys. Rev. Lett. 86 (2001) 2227.

13. G.W. Bennett, et al., (Muon $(g-2)$ Collaboration), Phys. Rev. Lett. 89, 101804 (2002)

14. G.W. Bennett, et al., (Muon $(g-2)$ Collaboration), Phys. Rev. Lett. 92, 161802 (2004).

15. A $(g-2)_{\mu}$ Experiment to \pm 0.2 ppm Precision D.W. Hertzog (co-spokesperson), B.L. Roberts (co-spokesperson), W.M. Morse (resident spokesperson) The proposal can be found at: http://g2pc1.bu.edu/ roberts/

16. K. Hagiwara, A.D. Martin, Daisuke Nomura, and T. Teubner, Phys. Lett. B557, 69 (2003), and Phys. Rev. D69 093003 (2004). and A. Höcker, ICHEP04, which can be found at http://ichep04.ihep.ac.cn/program.htm

17. See $\sigma_{\text {Had }}$ presentations at EPS2005, http://www.lip.pt/events/2005/hep2005/ click on "Talks" and "Tests of the SM"

18. J-PARC Letter of Intent L17: An Improved Muon (g-2) Experiment at J-PARC, Contact person: B.L. Roberts.

19. J.R. Ellis, et al., Phys. Rev. D71 (2005) 095007, and references therein.

20. A. Czarnecki, W.J. Marciano and K. Melnikov, AIP Conf. Proc. 435, p409, hep-ph/9801218

21. P. Paradisi, hep-ph/0505046 2

22. E.M. Purcell and N.F. Ramsey, Phys. Rev. 78, 807 (1950)

23. L. Landau, Nucl. Phys. 3, 127 (1957).

24. N.F. Ramsey Phys. Rev. 109, 225 (1958).

25. J. R. Ellis, J. Hisano, M. Raidal and Y. Shimizu, Phys. Lett. B 528, 86 (2002).

26. J. R. Ellis and M. Raidal, Nucl. Phys. B 643 229, (2002).

27. K.S. Babu, B. Datta, and R.N. Mohapatra, Phys. Rev. Lett.85, 5064 (2000).

28. J.L. Feng, K.T. Matchev .Y, Shadmi, Nucl. Phys. B 613, 366 (2001), and Phys. Lett. B555, 89 (2003).

29. J. Bailey, et. al, Nucl. Phys. B150, 1 (1979).

30. P.G. Harris, et al., Phys. Rev. Lett. 82, 904 (1999).

31. B.C. Regan, et al., Phys. Rev. Lett. 88, 071805-1 (2002).

32. J-PARC Letter of Intent L22, Search for a permanent muon electric dipole moment at the $10^{-24}$ e cm level., Y. Kuno, J.Miller, Y. Semertzidis spokespersons.

33. K. Jungmann at this meeting.

34. B. Pontecorvo, Zh. Eksp. Teor. Fiz. 33 (1957) 549, [Sov. Phys. JETP 6 (1958) 429]; G. Feinberg and S. Weinberg, Phys. Rev. 123 (1961) 1439.

35. L. Willmann, et al., Phys. Rev. Lett. 82 (1999) 49

36. P. Herczeg and R.N. Mohapatra, Phys. Rev. Lett. 69 (1992) 2475; A. Halprin, Phys. Rev. Lett. 48 (1982) 1313; R.N. Mohapatra, Z. Phys. C56 (1992) 117; G.G. Wong and W.S. Hou, Phys. Rev. D50 (1994) R2962; A. Halprin and A. Masiero, Phys. Rev. D48 (1993) 2987; H. Fujii, Y. Mimura, K. Sasaki and T. Sasaki, Phys. Rev. D49 (1994) 559; P.H. Frampton and M. Harada, Phys. Rev. D58 (1998) 095013; V. Pleitez, Phys. Rev. D61 (2000) 057903; G. Cvetic, et al., hep-ph/0504126

37. Shinya Kanemura at this meeting, and S. Kanemura, et al., Phys. Lett. B607 (2005) 165.

38. F.J.M. Farley, et al., Phys. Rev. Lett. 92, 052001 (2004).

39. J-PARC Letter of Intent L24: The PRISM Project - A Muon Source of the WorldHighest Brightness by Phase Rotation Contact persons: Y. Mori, K. Yoshimura, N. Sasao, Y. Kuno

40. F. Mulhauser, at this meeting. $\mu$ Lan experiment, D. Hertzog and R. Carey spokespersons, MuCap experiment, C. Petijean and P. Kammel spokespersons.

41. K. Shimomura, at this meeting.

42. E. Morenzoni, at this meeting.

43. K. Ishida, at this meeting.

44. A.Toyoda et al, Phys. Rev. Lett. 90 (2003) 243401 